\title{
On the origins of unsteadiness and three-dimensionality in a laminar separation bubble
}

\author{
By Vassilios Theofilis, Stefan Hein and Uwe Dallmann \\ Deutsches Zentrum für Luft- und Raumfahrt e.V. (DLR), \\ Institute of Fluid Mechanics, Transition and Turbulence, \\ Bunsenstraße 10, D-37073 Göttingen, Germany
}

\begin{abstract}
We analyse the three-dimensional non-parallel instability mechanisms responsible for transition to turbulence in regions of recirculating steady laminar two-dimensional incompressible separation bubble flow in a twofold manner. First, we revisit the problem of Tollmien-Schlichting (TS)-like disturbances and we demonstrate, for the first time for this type of flow, excellent agreement between the parabolized stability equation results and those of independently performed direct numerical simulations. Second, we perform a partial-derivative eigenvalue problem stability analysis by discretizing the two spatial directions on which the basic flow depends, precluding TS-like waves from entering the calculation domain. A new two-dimensional set of global amplified instability modes is thus discovered. In order to prove earlier topological conjectures about the flow structural changes occurring prior to the onset of bubble unsteadiness, we reconstruct the total flowfield by linear superposition of the steady two-dimensional basic flow and the new most-amplified global eigenmodes. In the parameter range investigated, the result is a bifurcation into a three-dimensional flowfield in which the separation line remains unaffected while the primary reattachment line becomes three dimensional, in line with the analogous result of a multitude of experimental observations.
\end{abstract}

Keywords: linear non-local instability; topological flow changes;

laminar separation bubble; global instability; structural instability

\section{Introduction}

The subjects of laminar flow separation, its linear and nonlinear instability and transition to turbulence have attracted experimental and theoretical interest for the best part of the last century. The understanding of the physics governing these phenomena, especially with respect to vortex shedding and its relation to instability mechanisms, is still far from being satisfactory, as witnessed by the plethora and diversity of contributions to this issue. Motivation for continuous study from an aerodynamic point of view is primarily offered by the formation of laminar separation bubbles in the neighbourhood of the mid-chord region of an aerofoil at moderate angles of attack and low Reynolds numbers. Under the influence of environmental excitation, the laminar separated flow has been demonstrated to undergo transition and reattach as turbulent flow in a multitude of experiments (see, for example, Dovgal 
et al. 1994) and numerical simulations (see, for example, Bestek et al. 1989). As in attached boundary-layer flows, essential features of the transition process are the flow unsteadiness and three dimensionality.

It should be noted that several parameters - such as Reynolds number, means of introduction, extent of application and strength of an adverse pressure gradientshould be taken into account in order for a laminar separation bubble to be characterized. Rather than relying on parametric studies, however, our concern is with the identification of the physical mechanisms underlying the onset of unsteadiness and three dimensionality of the flow. Specifically, we have the following questions in mind.

(i) Does the bubble only act as an amplifier of environmental disturbances entering the separated flow region, or does it also have the potential to generate unstable modes in the absence of incoming disturbances?

(ii) In either case, what are the structural changes experienced by the laminar separation bubble on account of linear instability mechanisms?

(iii) Further, how do these structural changes relate to the topological flow changes which have been conjectured in the literature as being responsible for the onset of vortex shedding from separation bubbles?

Abandoning any intention of offering a complete discussion here of the large body of work concerning instability and transition of flow encompassing a laminar separation bubble, we confine ourselves to drawing the demarcation line between the approaches used in the past and those presently utilized in order to address the above questions. We also stress that, rather than performing a study at Reynolds numbers relevant to industrial applications (as done, for example, by Spalart \& Coleman (1997)), we confine ourselves to low transitional Reynolds numbers.

We address the questions posed within the frame of linear instability analyses in which disturbances are treated as non-periodic two-dimensional functions of the chordwise and wall-normal spatial coordinates. Two complementary approaches have been followed. First, we consider slow development of the basic flow in the streamwise direction and apply our non-local analysis tools, based on the parabolized stability equations (PSE) developed by Hein et al. (1994). Second, we perform a partialderivative eigenvalue problem stability (PEPS) analysis (Theofilis 1998) in which both the chordwise and wall-normal directions are fully resolved. In both approaches the linear disturbances are taken to be periodic in the spanwise direction. It should be noted that we study solely instabilities of a convective nature when employing the PSE, since evidence exists (Allen \& Riley 1995) that breakdown to turbulence in basic flows with small separation bubbles, in terms of extent of the bubble compared with a typical length and in terms of magnitude of the steady recirculating flow compared with the freestream velocity, is of a convective nature.

In $\S 2$ we present the two basic flows utilized for our numerical linear instability experiments. Elements of the theories governing the non-local and global instability analyses based on the PSE and the PEPS, respectively, are presented in $\S 3$. In $\S 4$ we present a comparison of our PSE results with those of the spatial direct numerical simulations (DNS) of Rist \& Maucher (1994) and classic Orr-Sommerfeld theory. New global linear instability modes discovered by the PEPS analysis are also presented in $\S 4$. Section 5 is devoted to discussing our findings from a topological 
analysis point of view. Concluding remarks relating our results to earlier work are offered in $\S 6$.

\section{The basic flows}

The instability properties and characteristics of two different incompressible twodimensional flat-plate laminar boundary-layer flows with separation bubbles encompassed are analysed. In both cases laminar separation is caused by a decelerated freestream velocity $U_{\mathrm{e}}(x)$ prescribed at some fixed distance from the wall. The instabilities developing upon the first basic flow (BF1) were studied in a DNS by Rist \& Maucher (1994). They prescribed a smooth deceleration of $\Delta U_{\mathrm{e}}=0.09$ between $x_{1}=0.71$ and $x_{2}=2.43$, where $x$ and $y$ denote streamwise and wall-normal direction, respectively, and $(U, V)^{\mathrm{T}}$ denotes the corresponding velocity vector. Upstream of $x_{1}$ and downstream of $x_{2}$ the freestream velocity is kept constant. Flow quantities are made non-dimensional with the freestream velocity $U_{\infty}^{*}=30 \mathrm{~m} \mathrm{~s}^{-1}$ upstream of $x_{1}$ and a global reference length $L^{*}=0.05 \mathrm{~m}$. The global Reynolds number $R e=U_{\infty}^{*} L^{*} / \nu^{*}$ was set to $R e=10^{5}$. The steady basic flow was calculated by Rist \& Maucher (1994) using their DNS code with a Blasius solution as the inflow condition prescribed at $x_{0}=0.37$. The flow separates at $x_{\mathrm{S}} \approx 1.73$ and reattaches at $x_{\mathrm{R}} \approx 2.21$. Downstream of the bubble the flow asymptotically recovers to a Blasius boundary layer, as indicated by the shape factor $H_{12}$ (Hein et al. 1998).

Linear instability mechanisms supported by a second basic flow (BF2), that described by Briley (1971), have also been studied. This flow is calculated in two stages. First, the non-similar boundary-layer equations are solved subject to the classic linearly decelerating streamwise velocity component distribution due to Howarth (1938). Second, a two-dimensional incompressible DNS is performed using an efficient and spectrally accurate algorithm based on matrix diagonalization. The inflow boundary data for the DNS are provided by the boundary-layer solution taken at a location well before separation occurs. The imposition in the DNS of the same freestream velocity distribution as in Howarth's (1938) case up to a given downstream position, beyond which the freestream velocity is kept constant, results in the formation of a separated flow region which compares very well with those presented by both Briley (1971) and Cebeci \& Stewartson (1983). The parameters held constant were the Reynolds number, $R e=10^{6} / 48$, and the inflow location, $\xi_{\text {inf }}=0.05$ (corresponding to $x_{\mathrm{inf}}=\left(\beta_{0} / \beta_{1}\right) \xi_{\mathrm{inf}}=0.05 / 3$ ), while those varied were the downstream extent of the calculation domain, taking values from $\xi=0.5$ (Briley 1971) to $\xi=1.5$ (Cebeci \& Stewartson 1983), and the (scaled) location in the wall-normal direction where the pressure gradient is imposed, varying from $\eta=5.4$ in the former reference to $\eta=7.5$ in the latter reference.

One essential difference between BF 1 and BF2 is that, in the former basic flow, the Reynolds number based on the displacement thickness $\delta_{1}$ at $x_{1}=0.71$ is $\operatorname{Re}_{\delta_{1}} \approx$ 460. According to linear local instability theory, the critical Reynolds number of Blasius flow is $R e_{\delta_{1}}=420$ (see, for example, Schlichting 1979). Hence, for this basic flow, Tollmien-Schlichting instability sets in upstream of the position $x_{1}$, where flow deceleration starts. In BF2, on the other hand, the Reynolds number at the outflow boundary $\xi_{\text {out }}=1.5$, where a Blasius boundary layer is recovered, is $R_{\delta_{1}}=290$, well upstream of the Blasius critical $R e_{\delta_{1}}$. 


\section{Linear instability theories}

The non-local/global nature of the instability analyses used derives from the decomposition of a perturbed flowfield into a steady two-dimensional basic and an unsteady three-dimensional disturbance component according to

$$
\boldsymbol{Q}(x, y, z, t)=\boldsymbol{Q}_{\mathrm{b}}(x, y)+\varepsilon \boldsymbol{Q}_{\mathrm{p}}(x, y) \exp \mathrm{i} \Theta+\text { c.c. },
$$

with $\boldsymbol{Q}_{\mathrm{b}}=(U, V, 0, P)^{\mathrm{T}}$ indicating the real basic flow and $\boldsymbol{Q}_{\mathrm{p}}=(\hat{u}, \hat{v}, \hat{w}, \hat{p})^{\mathrm{T}}$ denoting complex-valued perturbations to it. Here, $x$ denotes the streamwise direction, $y$ the wall-normal direction and $z$ the spanwise spatial direction; the imaginary unit is $\mathrm{i}=\sqrt{ }-1$ and c.c. denotes complex conjugation in order for the total field to remain real. Linearization about $\boldsymbol{Q}_{\mathrm{b}}$ follows, based on the argument of smallness of $\varepsilon$ and the basic flow terms, themselves satisfying the Navier-Stokes and continuity equations, are subtracted out. The difference between the two approaches stems from the ansatz made for the complex function $\Theta$, as follows.

\section{(a) Non-local analysis based on the PSE}

The linear non-local instability analyses were performed with the NOnLOcal Transition analysis (NOLOT)/PSE code developed in cooperation between DLR and Flygtekniska Forsokanstalten (Hein et al. 1994). $\dagger$ In the NOLOT/PSE analysis, both $\boldsymbol{Q}_{\mathrm{b}}$ and $\boldsymbol{Q}_{\mathrm{p}}$ are functions of $y$ slowly varying in $x$; we call this approach a non-local analysis, as opposed to the classic local theory in which both $\boldsymbol{Q}_{\mathrm{b}}$ and $\boldsymbol{Q}_{\mathrm{p}}$ are functions of $y$ alone. The wave function

$$
\Theta=\int_{x_{0}}^{x} \alpha(\xi) \mathrm{d} \xi+\beta z-\Omega t,
$$

with $\alpha$ a slowly varying streamwise wavenumber, is intended to capture practically all oscillatory-type streamwise variations of the disturbance, that is the variations on the fast scale. The disturbances are assumed to grow in the streamwise direction $x$ only, and spatially evolving wave-like instabilities are considered. Hence, the streamwise wavenumber $\alpha$ is represented by a complex quantity, whereas the spanwise wavenumber $\beta$ and the circular frequency $\Omega$ are real parameters.

Since both the amplitude function and the wave function depend on the $x$-direction, a normalization condition is required which removes this ambiguity, as introduced by Bertolotti et al. (1992):

$$
\int_{0}^{y_{e}} \boldsymbol{Q}_{\mathrm{p}}^{\dagger} \frac{\partial \boldsymbol{Q}_{\mathrm{p}}}{\partial x} \mathrm{~d} y=0 .
$$

The superscript ${ }^{\dagger}$ refers to the complex conjugate and $y_{\mathrm{e}}$ stands for the upper boundary of the discretized domain. The normalization condition is used as an iteration condition in order to ensure that as much as possible of the streamwise variation of the disturbance is transferred into the wave function. The remaining part of the streamwise variations is described by the amplitude functions and is small; consequently, second derivatives of the amplitude functions in $x$ are discarded. This leads

$\dagger$ For the purposes of the present study the code was run in the incompressible limit at which excellent agreement with results obtained by incompressible codes has been shown by Hein et al. (1994). 
to a system of partial differential equations of the form

$$
A \boldsymbol{Q}_{\mathrm{p}}+B \frac{\partial \boldsymbol{Q}_{\mathrm{p}}}{\partial y}+C \frac{\partial^{2} \boldsymbol{Q}_{\mathrm{p}}}{\partial y^{2}}+D \frac{\partial \boldsymbol{Q}_{\mathrm{p}}}{\partial x}=0 .
$$

Details of the consistent multiple-scales approach based on a Reynolds number expansion used to derive the non-local stability equations, which are closely related to the PSE (Bertolotti et al. 1992; Herbert 1997), and the elements of the matrices $A$, $B, C, D$ can be found in Hein et al. (1994). They represent an initial-boundary-value problem that can be solved efficiently by a marching procedure in the $x$-direction with upstream effects neglected, an approach valid for convectively unstable flows only. The partial differential equations system (3.4) is discretized by a first-order backward Euler scheme in the $x$ spatial direction and fourth-order compact finite differences in the $y$ spatial direction. On the amplitude functions $(\hat{u}, \hat{v}, \hat{w})$ we impose homogeneous Dirichlet boundary conditions at the wall $(y=0)$ and asymptotic boundary conditions enforcing exponential decay at the upper boundary $y_{\mathrm{e}}$ (Hein $e t$ al. 1994). Initial conditions are provided by a local parallel instability analysis using the same code, with the matrix $D$, all streamwise derivatives in matrices $A, B$ and $C$ and the wall-normal velocity component $V$ of the basic flow set to zero.

The spatial disturbance growth rate $\sigma$ in local instability theory is described by the negative imaginary part of the complex wavenumber $\alpha$, i.e. $\sigma=-\alpha_{i}$, whereas in non-local theory there is an additional contribution from the amplitude functions and various reasonable alternative definitions are possible; here we use either

$$
\sigma_{E}=-\alpha_{\mathrm{i}}+\frac{\partial}{\partial x} \ln (\sqrt{ } E), \quad \text { with } E=\int_{0}^{y_{\mathrm{e}}}\left(\|\hat{u}\|^{2}+\|\hat{v}\|^{2}+\|\hat{w}\|^{2}\right) \mathrm{d} y
$$

or

$$
\sigma_{u}=-\alpha_{\mathrm{i}}+\operatorname{Re}\left(\frac{1}{\hat{u}_{\mathrm{m}}} \frac{\partial \hat{u}_{\mathrm{m}}}{\partial x}\right)
$$

where $\hat{u}_{\mathrm{m}}$ denotes the value of $\hat{u}$ at the wall-normal coordinate where $\|\hat{u}\|$ reaches its maximum.

\section{(b) The partial derivative eigenvalue problem}

The assumptions of slow growth of $\boldsymbol{Q}_{\mathrm{b}}$ and $\boldsymbol{Q}_{\mathrm{p}}$, inherent in the PSE, may be relaxed by fully resolving both the streamwise and the wall-normal spatial directions; on account of this property, we term the PEPS a global instability analysis. In the temporal framework, as considered here, the complex function $\Theta$ becomes simply

$$
\Theta=\beta z-\Omega t
$$

where $\beta$ is a real wavenumber parameter and $\Omega$ is the sought complex eigenvalue whose real part indicates frequency and whose positive imaginary part is the growth rate in time $t$ of global disturbances. This definition of $\Theta$ suggests the second essential difference between the PSE and the PEPS approaches, namely that any structure that an eigenmode may have in the streamwise direction $x$, including that of a wavelike disturbance considered by the PSE, will be captured by $\boldsymbol{Q}_{\mathrm{p}}$ in (3.1).

Numerical aspects of the solution of the two-dimensional partial derivative eigenvalue problem are discussed by Theofilis (1998). Compared with that work, we have 
implemented here a novel version of the algorithm that applies to flow problems in which the wavenumber vector is perpendicular to the plane on which the basic flow is defined (Theofilis 2000). Specifically, straightforward redefinitions of the spanwise disturbance velocity component $\hat{w}$ and the eigenvalue $\Omega$ by i $\hat{w}$ and i $\Omega$, respectively, result in a disturbance flow system with real coefficients that, compared with the full problem, requires approximately half the storage and runtime to solve. The real non-symmetric generalized eigenvalue problem for the determination of $\Omega$ and $\boldsymbol{Q}_{\mathrm{p}}$ which results is as follows:

$$
\begin{aligned}
{\left[\mathcal{L}-\left(\mathcal{D}_{x} U\right)\right] \hat{u}-\left(\mathcal{D}_{y} U\right) \hat{v}-\mathcal{D}_{x} \hat{p} } & =\Omega \hat{u}, \\
-\left(\mathcal{D}_{x} V\right) \hat{u}+\left[\mathcal{L}-\left(\mathcal{D}_{y} V\right)\right] \hat{v}-\mathcal{D}_{y} \hat{p} & =\Omega \hat{v}, \\
\mathcal{L} \hat{w}-\beta \hat{p} & =\Omega \hat{w}, \\
\mathcal{D}_{x} \hat{u}+\mathcal{D}_{y} \hat{v}-\beta \hat{w} & =0,
\end{aligned}
$$

where

$$
\mathcal{L}=\left(\frac{1}{R e}\right)\left(\mathcal{D}_{x}^{2}+\mathcal{D}_{y}^{2}-\beta^{2}\right)-U \mathcal{D}_{x}-V \mathcal{D}_{y}, \quad \mathcal{D}_{x}=\frac{\partial}{\partial x} \quad \text { and } \quad \mathcal{D}_{y}=\frac{\partial}{\partial y} .
$$

For its solution we use spectral collocation on a rectangular Cartesian grid. The boundary conditions imposed are the straightforward viscous boundary conditions on all disturbance velocity components at the wall and compatibility conditions for the pressure; in the freestream the disturbance velocity components and disturbance pressure are required to decay. In an attempt to preclude disturbances from entering the recirculating flow area from upstream, homogeneous Dirichlet boundary conditions are imposed at the inflow. At the outflow boundary, linear extrapolation of all disturbance quantities from the interior of the integration domain is performed.

\section{Results}

(a) Short chordwise-wavelength instabilities studied by the PSE

A classic means of linear analysis of short-wavelength instabilities in laminar separation bubbles is based on local theory, in which the parallel flow assumption is made and the Orr-Sommerfeld equation (OSE) is solved (Allen \& Riley 1995). There is no rational approach leading to the latter equation that ignores both upstream influence and downstream development of the basic flow; nevertheless, both the OSE and spatial DNS predict, in close agreement, an explosive growth of convective instabilities entering the laminar separated flow (Bestek et al. 1989). On the other hand, an instability analysis approach which takes into account downstream development of the flow is based on the PSE. Due to the backflow within a separation bubble one might suspect that there is a significant amount of information travelling upstream and a marching procedure, as used in non-local instability analyses, is not appropriate. However, there is strong indication from both experiment and DNS that thin laminar separation bubbles are convectively unstable to short-wavelength disturbances of small amplitude initiated upstream of the bubble (Dovgal et al. 1994; Rist et al. 1996). This encourages us to embark upon application of PSE for this type of flow.

As a first basic flow we take BF1 of Rist \& Maucher (1994). They compared the results of linear local theory with those of their DNS, the latter run at low 


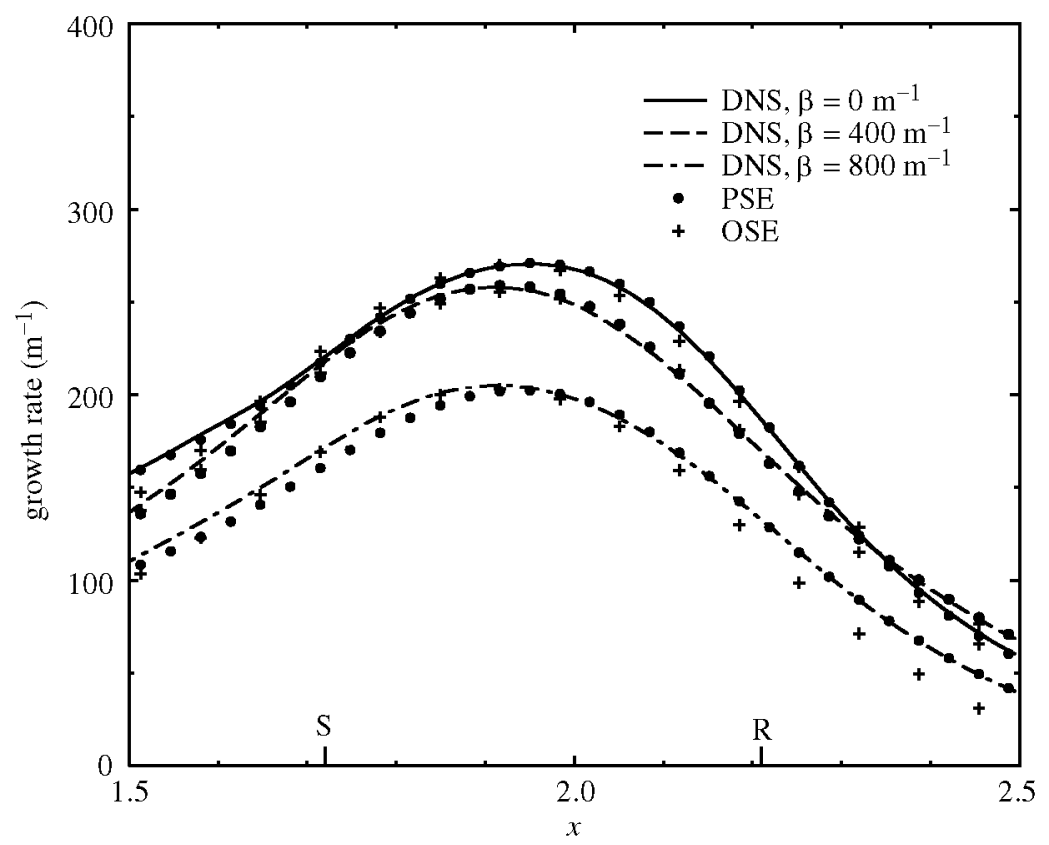

Figure 1. Comparison of non-local growth rates, $\sigma_{u}$, for BF1 obtained by NOLOT/PSE with the DNS data of Rist \& Maucher (1994) and our OSE solutions. Separation and reattachment are denoted by 'S' and ' $R$ ', respectively.

disturbance amplitudes; we use their growth rate data as a reference. Figure 1 shows the disturbance growth rates for $f \approx 1719 \mathrm{~Hz}$ (reduced frequency $F=1.8 \times 10^{-4}$ ) and three different (dimensional) spanwise wavenumbers $\beta=0,400$ and $800 \mathrm{~m}^{-1}$. The corresponding wave angles $\phi$ at separation point $x_{\mathrm{S}}$ are $\phi \approx 0,22$ and $41^{\circ}$. The linear local (OSE) and the non-local (PSE) results are compared with those obtained in the DNS of Rist \& Maucher (1994). The non-local growth rate is defined as in the DNS, i.e. on the maximum streamwise disturbance velocity (3.6).

The result of major significance in this figure is the agreement between the linear PSE and DNS results, which is found to be influenced by the wave angle. For twodimensional waves PSE delivers instability results that are in excellent agreement with those obtained by DNS at all $x$ positions, with the obvious advantage that PSE results are obtained at orders-of-magnitude lower computing effort compared with DNS. The agreement is still very convincing for three-dimensional waves, although a discrepancy between PSE and DNS results that increases with wave angle is to be found upstream of the bubble. There are at least three possible explanations for this wave-angle dependent discrepancy. First, it might be thought that the upstream influence neglected in PSE might cause PSE results to deviate from those of the DNS. Second, violation of the PSE assumption of a weakly non-parallel flow in the neighbourhood of the separation point may be considered to be responsible for the discrepancy. Third, transients in the DNS data may cause the small differences. The first reason cannot explain the discrepancies because these are present upstream of separation, in a region of attached boundary-layer flow where upstream effects are indeed negligible but disappear in the second half of the bubble, despite the existing backflow. In order for the second reason to be verified, one should refer to 

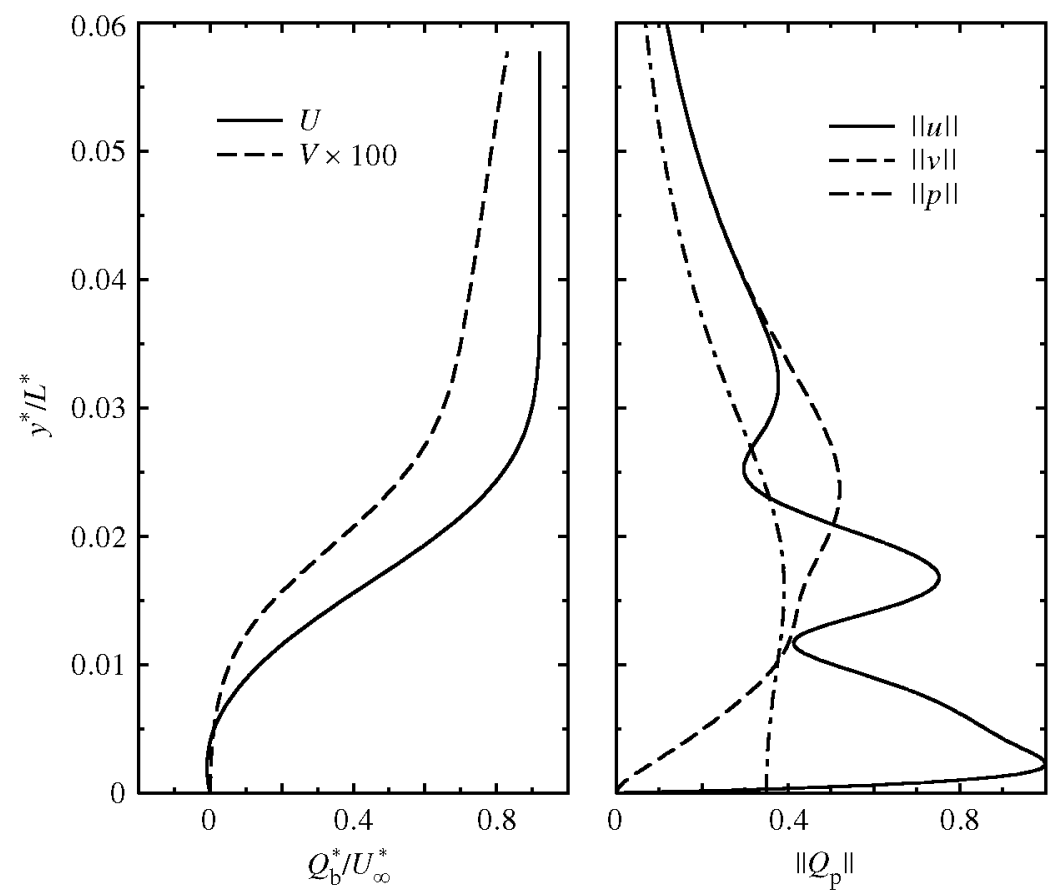

Figure 2. Velocity profiles of the laminar basic flow BF1 and the amplitude functions of a two-dimensional wave of $f=1719 \mathrm{~Hz}$ at $x=1.984$ within the recirculating flow.

the magnitude of the non-parallel terms in the attached, decelerating part of the flow domain compared with that of the non-parallel terms in the recirculating zone.

We believe that the third reason plays an essential role, since disturbances are generated in the DNS by periodic blowing and suction at the wall, immediately upstream of the region where flow deceleration is enforced. It therefore takes some downstream distance until these artificially introduced disturbances are converted into TS-like instabilities whose growth rate is no longer affected by the actual means of their generation. On the other hand, memory effects are shorter in our PSE analyses, which were started with TS waves (solutions of the OSE equation) imposed at the inflow boundary; the distance from the end of the suction strip to $x=1.5$ corresponds to less than six disturbance wavefronts. It is well known that both the effects of flow non-parallelism and the effects of disturbance history increase with disturbance wave angle. If, as we think, the differences are indeed caused by remaining transients, they should be more pronounced at smaller values of $x$. However, this could not be verified, because DNS growth-rate data for $x<1.5$ are not available from Rist \& Maucher (1994). These authors also performed an OSE analysis (not presented here), the results of which compare very well with our linear local analysis (also shown in figure 1). The agreement between our OSE growth rate results and DNS is quite close, in line with the findings of Bestek et al. (1989) and others. However, the agreement between OSE and DNS can be seen to be much inferior to that between PSE and DNS results.

Note that the largest growth rates are of the same order of magnitude as the disturbance wavenumbers, which means that the disturbances grow much more rapidly 

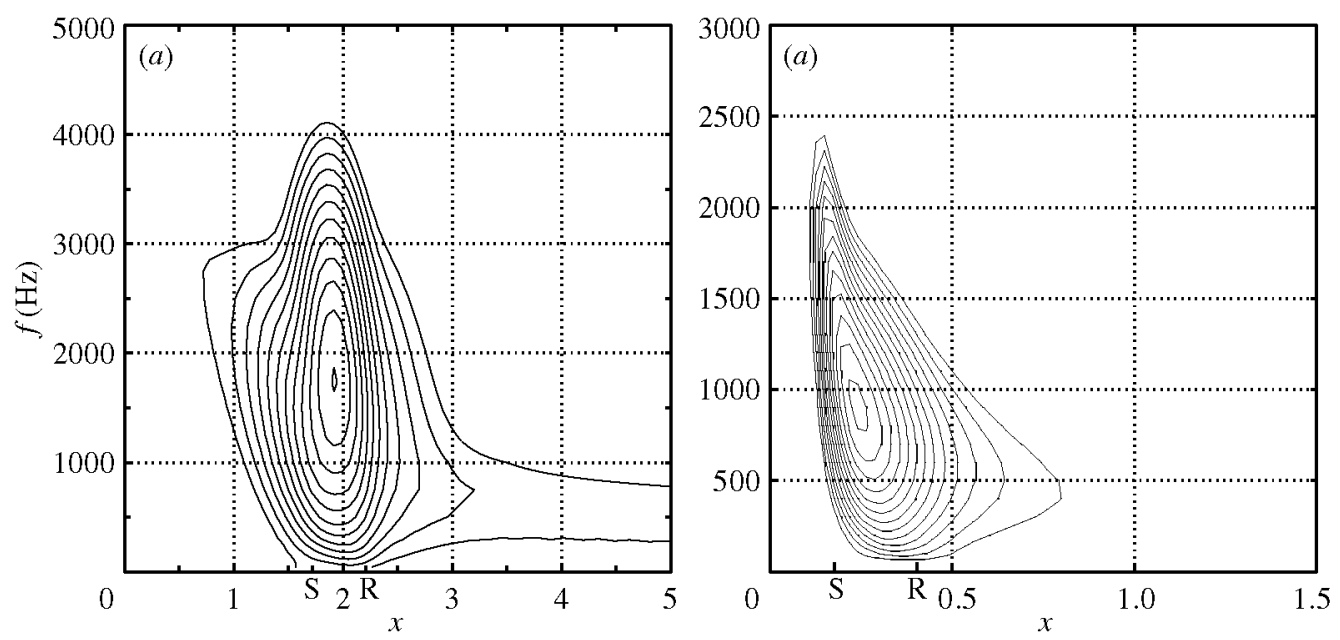

Figure 3. Isolines of constant non-local growth rate $\sigma_{E}$ for BF1 (a) and BF2 (b). Frequencies $f$ of unstable waves against scaled downstream distance are plotted. The outermost curves correspond to neutral stability and the increments in contour lines are $\delta \sigma_{E}=25$ and 10 for BF1 and $\mathrm{BF} 2$, respectively.

than those in Blasius flow. The non-local amplitude functions of the two-dimensional wave of $f \approx 1719 \mathrm{~Hz}$ are plotted in figure 2 for the downstream position $x=1.984$, alongside those of the corresponding velocity profiles of the basic flow. $\hat{u}$ has maxima inside the backflow region close to the wall, at the inflection point of the streamwise velocity profile of the basic flow and at the edge of the separated boundary layer, while $\hat{v}$ is rather large. Since the two-dimensional wave is the most amplified at almost all downstream positions (as seen in figure 1), we focus all subsequent NOLOT/PSE studies on two-dimensional waves. The instability diagram for twodimensional short-wavelength disturbances is shown in figure 3. Isolines of constant non-local growth rate (defined by 3.5 ) are plotted; the outermost isoline represents the curve of neutral stability. This growth-rate definition was chosen because in the separated flow region the disturbance amplitude functions have several maxima in the wall-normal direction (as seen in figure 2). The instability diagram for BF2 is also shown in figure 3 . Its general shape appears different from that of BF1, the latter having an instability region of the decelerating and separated flow embedded into the region of TS instability, unlike BF2 where the two are clearly separated (see also comments in $\S 2$ ).

\section{(b) Global disturbances addressed by the PEPS}

In the previous section we focused on instabilities of an oscillatory nature, the streamwise length-scale of which is small compared with that of the basic flow; this structure is exploited by the ansatz (3.2), and efficient marching procedures make a NOLOT/PSE approach the method of choice for accurate results to be delivered within reasonable computing time for this type of instability. On the other hand, an analysis of disturbances developing over length-scales comparable with that of the basic flow using NOLOT/PSE may deliver inaccurate results. Short of resorting to a spatial DNS in order to take both upstream influence and downstream 


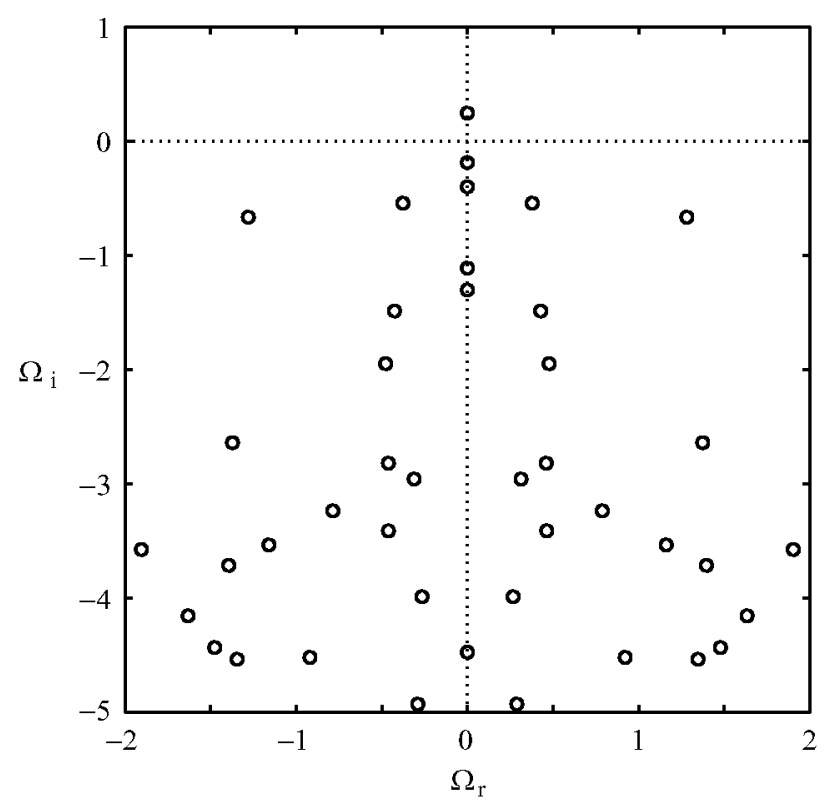

Figure 4. The window of the spectrum of global PEPS disturbances in the neighbourhood of $\Omega=0$.

development into account, disturbances having length-scales comparable with that of the basic flow are best analysed using numerical solutions of the partial derivative eigenvalue problem in which the available computing resources are devoted to the simultaneous resolution of both the $x$ and $y$ spatial directions. We concentrate here on presenting results for the BF 2 basic flow, obtained using the pressure-gradient parameters $\beta_{0}=100$ and $\beta_{1}=300$ at $R e=10^{6} / 48$. The eigenvalue spectrum in the neighbourhood of $\Omega=0$ and for a spanwise wavenumber $\beta=20$ is shown in figure 4. Stationary $(\operatorname{Re}\{\Omega\}=0)$ as well as travelling $(\operatorname{Re}\{\Omega\} \neq 0$ ) modes are to be found in this window of the spectrum, calculated by the Arnoldi algorithm (Theofilis 1998). The travelling modes appear in symmetric pairs, indicating that there is no preferential direction in $z$.

At this set of parameters, the most unstable mode is a stationary disturbance; $\uparrow$ the eigenvector $\boldsymbol{Q}_{\mathrm{p}}$ of this mode is presented in figure 5 . In order to aid the discussion on this figure, the position of the laminar basic flow separation bubble is indicated by a dotted line. From a numerical point of view, we stress that our results show that no effect of the downstream boundary conditions can be found in these PEPS solutions, a result which we attribute to a novel treatment of the pressure boundary conditions, which will be presented elsewhere. It suffices to mention here that the same boundary conditions were shown to perform equally well in another opensystem problem solved, that in the infinite swept attachment-line boundary layer (Lin \& Malik 1996; Theofilis 1997). Most of the activity in all disturbance eigenfunctions is confined within the boundary layer, with the neighbourhood of the inflow region being innocuous, as imposed by the inflow boundary condition. Significantly,

$\dagger$ A standing-wave pattern results from the linear superposition of the symmetric pairs of travelling modes. 

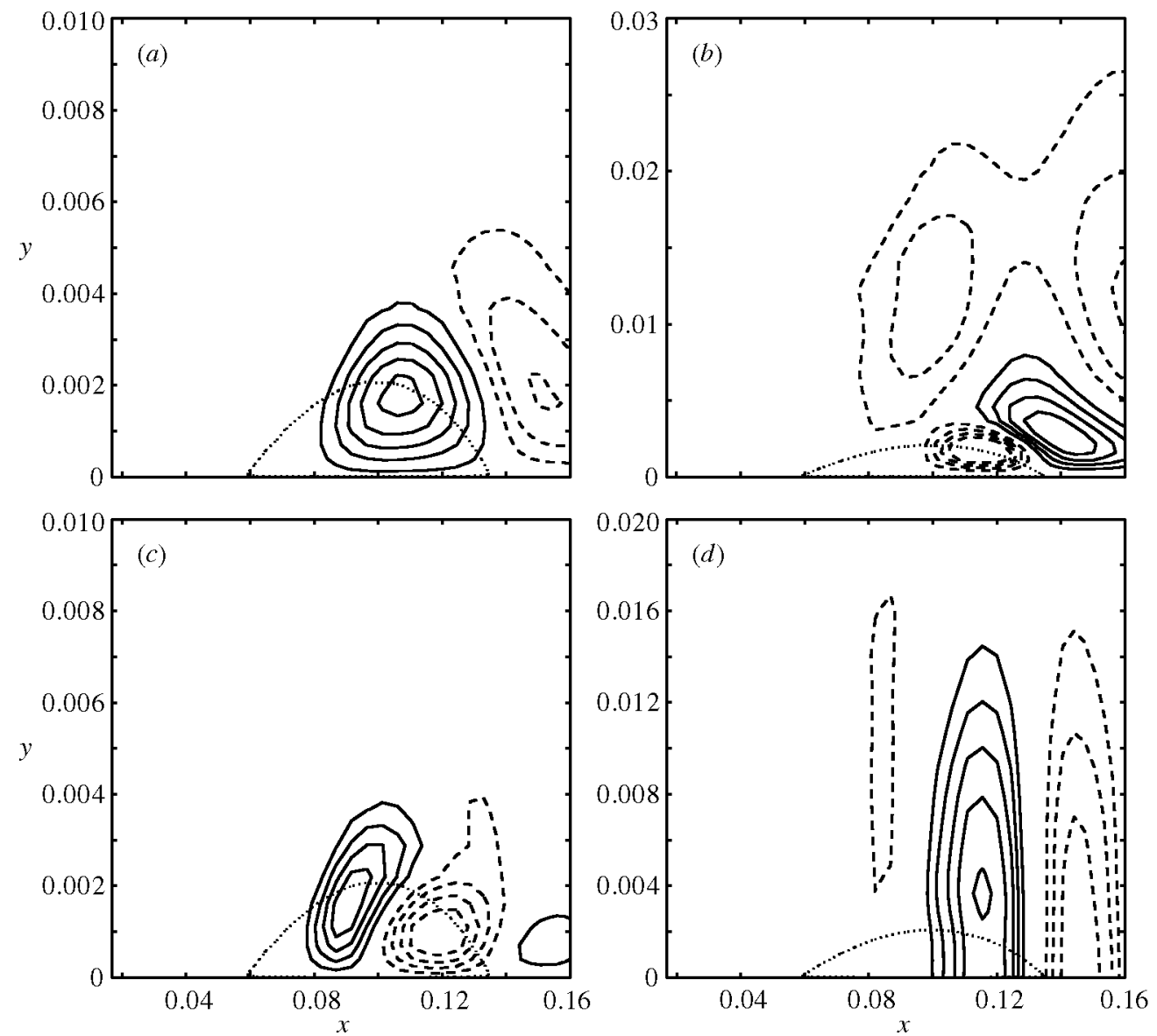

Figure 5. Normalized disturbance velocity components and pressure distribution of the unstable stationary global mode in figure 4 . Contour levels to be read as outer-to-inner values, where available. (a) $\operatorname{Re}\{\hat{u}\} ; 0.1(0.2) 0.9$ (solid), $-0.1(-0.1)-0.3$ (dashed). (b) $\operatorname{Re}\{\hat{v}\} ;-0.2(-0.2)-0.8$ (solid), 0.2(0.2)0.8 (dashed). (c) $\operatorname{Re}\{\hat{w}\} ; 0.2(0.2) 0.8$ (solid), $-0.2(-0.2)-0.8$ (dashed). (d) $\operatorname{Re}\{\hat{p}\}$; $0.2(0.1) 0.6$ (solid), $-0.2,-0.3,-0.4$ (dashed). The phase of this eigenvector $\theta(x, y)$ is approximately constant: $\theta \approx \pi$. In all results the location of the laminar separation bubble is indicated by the dotted line. Note the different $y$-scales in the different eigenvectors.

the neighbourhood of the basic laminar flow separation point is also unaffected, as is clearly demonstrated by the level of activity of all disturbance velocity components and pressure in that region. The peak of both the chordwise and the wall-normal linear disturbance velocity components is to be found just downstream of the maximum extent of the primary recirculation region, $x \approx 0.1$, with lower-level linear activity continuing past the point of primary reattachment. Interestingly, $\hat{w}$ (which is the only source of three dimensionality in this linear framework) is mostly distributed within the primary separation bubble and has a tendency to split the latter into two regions of fluid moving in opposite directions. Pressure also has an interesting signature, with almost purely sinusoidal disturbances generated within the primary laminar bubble and persisting well after the laminar basic flow has reattached. 
(a)

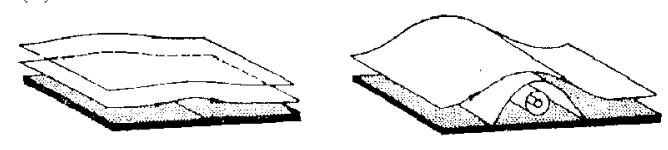

(b)
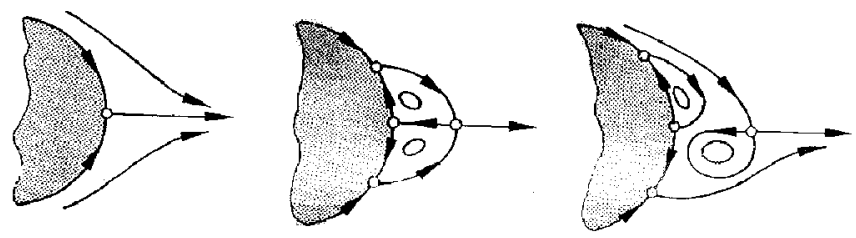

Figure 6. Local topological changes of flow structures associated with the formation of separation bubbles, and global topological change associated with the onset of vortex shedding behind a blunt body.

(a)
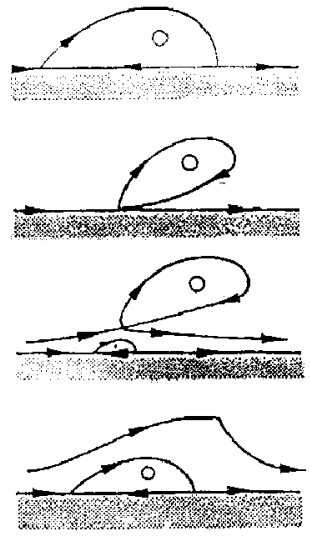

(b)
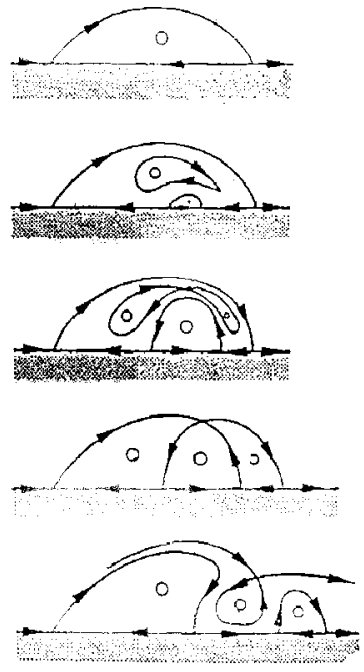

Figure 7. Two different conjectures $((a)$ and $(b))$ for topological changes of a separation bubble's structure associated with the onset of vortex shedding.

\section{Topological conjectures for structural separation-bubble instability}

A separation bubble appears as a region of recirculating flow between a separation point and a point of flow attachment located at a wall (figure $6 a$ ). $\dagger$ These two points form half-saddles in a cross-sectional plane that is parallel to the streamlines. At the wall, the locus of these points creates separation and reattachment lines, respectively, where a direction reversal of the near-wall flow occurs since the wall-shear stress changes its sign. The single streamline (streamsurface) that connects these

$\dagger$ Figures 6, 8, 10 and 11 are also available at http://www.sm.go.dlr.de/sm-sm_info/TRTinfo/ gallery.htm. 

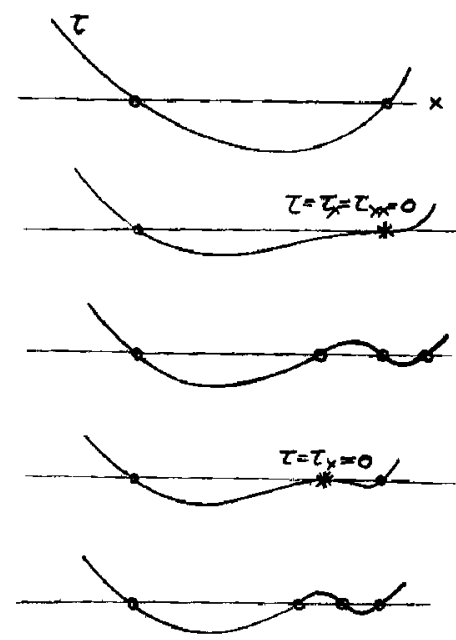

Figure 8. Generic wall-shear stress distributions associated with the formation of multiple-structured separation bubbles. The asterisks indicate degenerate critical points for local topological changes.

two 'critical' points (lines) and the wall itself define a boundary of the region of separated, recirculating fluid, which is characterized, additionally, by a centre point of a 'vortex' (Dallmann et al. 1997). Hence, at incipient separation (not covered by our present investigation), the two half-saddles and the centre point are created simultaneously at the wall, and a separation bubble and a 'vortex' are created via local structural (topological) changes of a flow. In the structurally stable regime, the simple bubble changes shape and grows in size but does not change its streamline topology. However, the topological structure of the pressure field will, in general, change at a different set of critical parameters, and this in turn can initiate a change in the bubble structure. A more complicated, but nevertheless local, structural flow change occurs at the onset of flow separation behind a blunt body (figure $6 b$ ). In twodimensional flows around blunt bodies it is well known that the onset of vortex shedding is associated with a break-up of saddle connections. How can a simple bubble become unsteady? One conjecture might be that due to a hydro/aerodynamic instability a region of recirculating fluid might be instantaneously swept downstream in a way sketched in figure $7 a$, such that the points of separation and attachment merge and release a patch of circulating fluid to convect downstream, while a new bubble forms at the wall. Only local structural flow changes are associated with this type of bubble unsteadiness and vortex shedding. Dallmann et al. (1995) provide numerical evidence for the correctness of an earlier topological conjecture, namely that, prior to unsteadiness and/or vortex shedding, multiple recirculation zones appear within a separation bubble and finally lead to a global structural flow change with multiple structurally unstable saddle-to-saddle connections. The separation line could then stay almost stationary despite the vortex shedding present, as sketched in the sequence of instantaneous patterns of figure $7 \mathrm{~b}$.

There are several routes leading from a simple generic bubble to a multiplestructured one before unsteadiness and vortex shedding sets in. In the most simple multiple-structured bubble case with several recirculating flow regions, the generic 


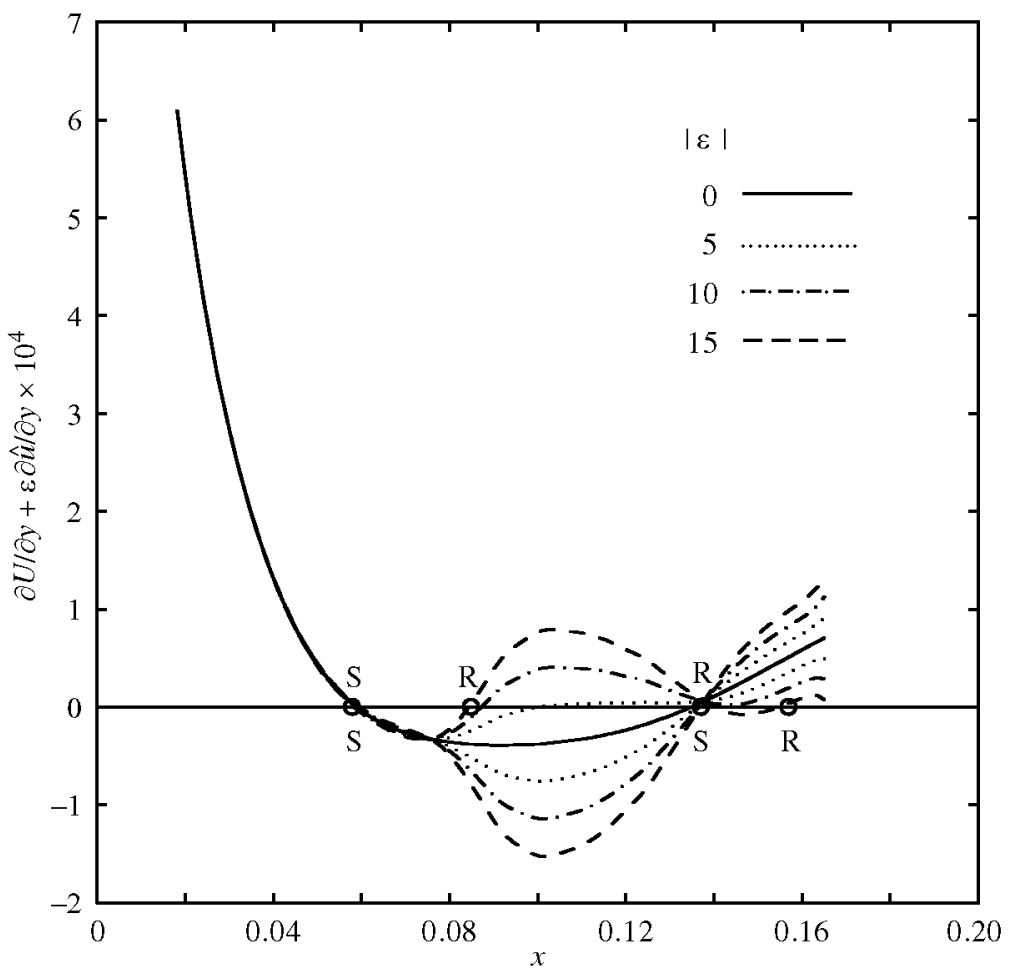

Figure 9. Wall-shear distribution due to the basic and different linear amounts of the disturbance streamwise velocity component. Shown are the separation (S) and reattachment (R) points as generated by the presence of the two-dimensional linear disturbance.

structure of the associated wall-shear stress will be of the kind sketched in figure 8 . The so-called singularity or degeneracy in the wall-shear stress graph (which appears where its magnitude together with first and, possibly, higher streamwise derivatives vanish) indicates a local degeneracy where a secondary bubble may form. In figure 9 the wall-shear pattern set up by the linear superposition of the basic flow and a linearly small amount of the streamwise disturbance velocity component $\hat{u}$ shown in figure 5 is shown; the latter amount is indicated on the figure in percentage terms. The effect of the presence of the stationary unstable mode on the primary separation and reattachment points may be seen in this figure. While the separation line (in three dimensions) remains unaffected in the presence of a linearly unstable global mode, the reattachment point of the basic flow at sufficiently high amplitudes of the linearly unstable mode becomes a secondary separation point, while both upstream and downstream of this point, secondary reattachment is generated linearly.

Let us now consider the onset of three dimensionality in a simple two-dimensional separation bubble as sketched in figure $10 . \dagger$ The separation and attachment lines of the two-dimensional flow (upper and middle parts of figure 10) will always be structurally unstable against any three-dimensional (steady or unsteady) perturbation.

$\dagger$ The mathematical framework of a description of the structural changes associated with local and (instantaneous) global topological flow changes and the set of 'elementary (three-dimensional) topological flow structures' which can appear at a rigid wall has been given by Dallmann (1988). 


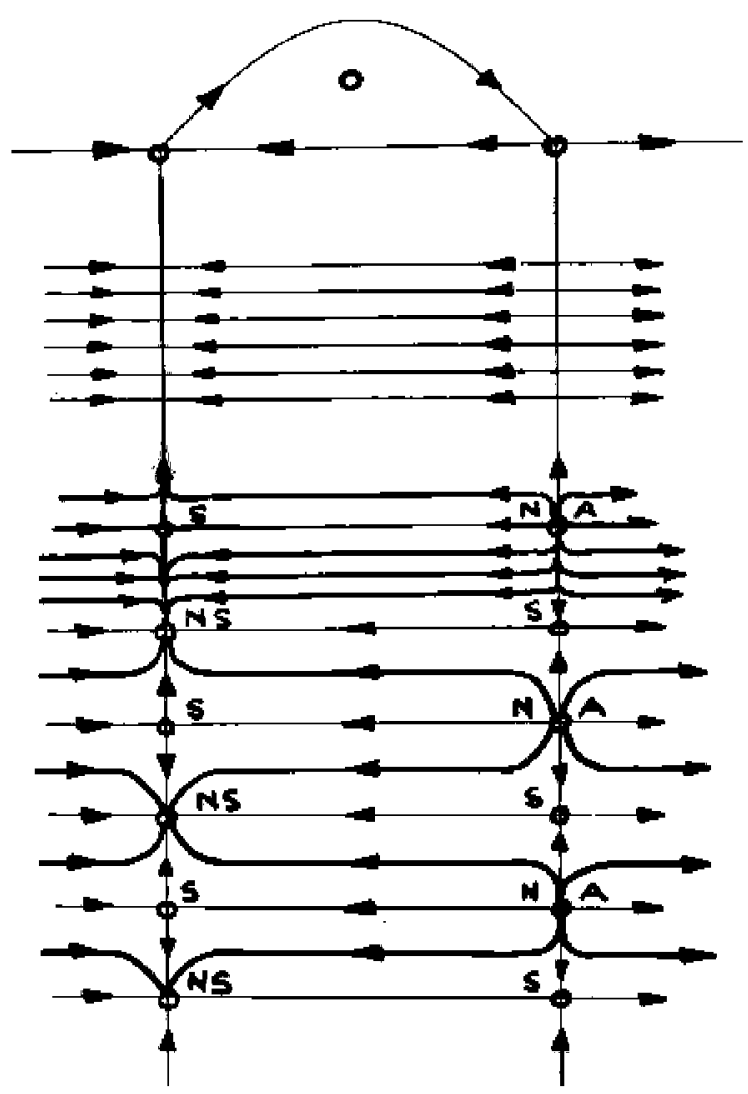

Figure 10. Streamline topology of a simple generic separation bubble (upper). Wall-flow pattern for a two-dimensional simple separation bubble (middle). Wall-flow pattern for a three-dimensional separation 'bubble' (lower).

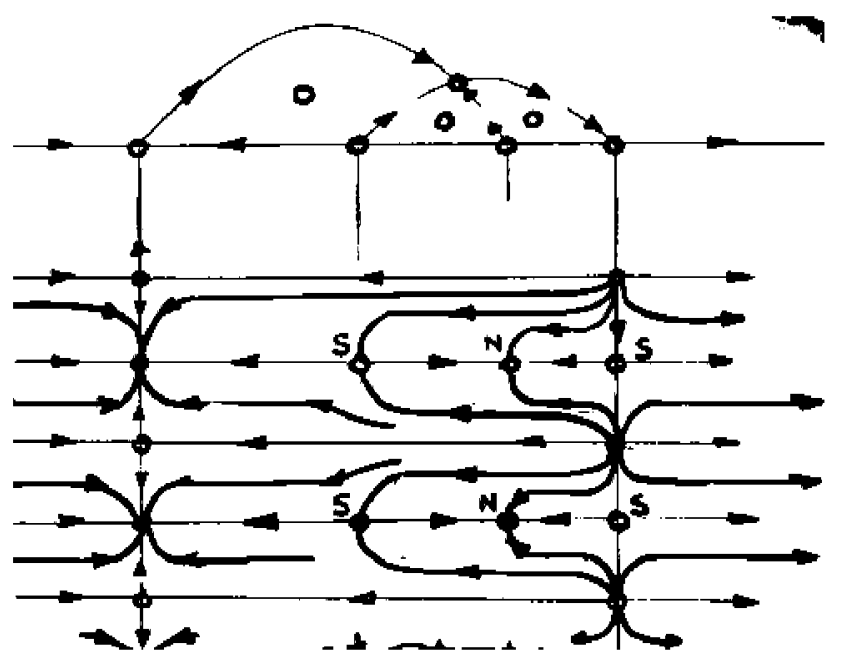

Figure 11. Streamline topology of a multiple-structured separation bubble (upper). Wall-flow pattern for the three-dimensional multiple-structured separation bubble (lower). 
The structurally stable configuration will be formed via a sequence of saddle and nodal points (either nodes or foci) along the separation as well as the attachment line(s). The resulting wall-streamline pattern (lower part of figure 10) does not necessarily differ much from the wall-flow pattern for a two-dimensional flow; however, 'three dimensionality' will always be observed close to the critical points, i.e. close to points of separation and/or attachment. What will be the effect of three-dimensional disturbances on the above-discussed incipient structural change from a simple twodimensional bubble into a multiple-structured one (which may appear and exist as a steady flow structure prior to flow unsteadiness and vortex shedding!)? For simplicity let us assume that the disturbance flow is periodic in the spanwise direction (as assumed in the PEPS and NOLOT/PSE analyses). The additional amplification of a wall-shear stress of the disturbance flow will increase and reduce the local mean-flow wall-shear stress in a spanwise-periodic way. The result may be found in figure 8, where the singularities in the wall-shear stress distribution (along $x$ ) will be structurally stabilized with additional critical points of attachment and separation occurring at one spanwise location, while the simple bubble structure will be preserved at other spanwise locations. Hence, the $x$ spatial oscillatory wall-shear stress behaviour (figure 8) of a spanwise-periodic disturbance flow of sufficient amplitude must immediately lead to a pronounced three-dimensional wall-flow pattern if a multiple-structured bubble occurs (upper part of figure 11). The above-presented results of the PEPS instability analysis clearly support these topological conjectures, namely the growth of a stationary disturbance mode leads to a multiple-structured separation 'bubble' prior to flow unsteadiness and vortex shedding. However, such a 'bubble' breaks up into a structure with complex three-dimensional topology due to the inherent three-dimensional character of the stationary spanwise-periodic disturbance mode. Three dimensionality will leave its footprints in the wall-flow pattern especially within the 'reattachment zone', i.e. where secondary 'bubbles' form.

\section{Discussion and outlook}

In this paper we subject a steady laminar two-dimensional boundary-layer flow that incorporates a recirculation zone to two distinct linear instability analyses and report two sets of significant findings. First, we apply PSE in order to study instability of a wall-bounded flow that encompasses a separation bubble. The agreement between PSE and spatial DNS results ranges from very good for three-dimensional disturbances to excellent for plane instability waves. Pauley et al. (1990) performed twodimensional DNS and reported that the Strouhal number $S t=f \delta_{2, \mathrm{~S}} / U_{\mathrm{e}}$ based on the vortex-shedding frequency $f$, the momentum thickness $\delta_{2}$ and the boundarylayer edge velocity $U_{\mathrm{e}}$ at separation is $S t=0.00686 \pm 0.6 \%$, and is independent of Reynolds number and pressure gradient; this result corresponds to dimensional frequencies of $f=1180 \mathrm{~Hz}$ and $f=986 \mathrm{~Hz}$ for BF1 and BF 2, respectively. Both match the most unstable frequencies found in our non-local instability analyses very well, as can be seen in figure 3. This finding is in line with those of Bestek et al. (1989), who reported that the mechanism of unsteadiness of separation bubbles is closely linked to the growth of disturbances travelling through the bubble. The agreement between PSE and DNS indicates that the upstream influence within a laminar separation bubble is indeed negligible for the short-wavelength disturbances considered, as long as the prevailing instabilities are of a convective nature. However, the extremely 
large disturbance growth rates in flows with laminar separation renders nonlinear PSE analyses much more challenging than in attached boundary layers.

The second significant finding presented in this paper concerns the existence of global instability modes inaccessible to local analyses. Solutions of the partial derivative eigenvalue problem have been discovered that correspond to both stationary and travelling global instabilities, periodic in the spanwise $z$-direction. While we present solutions at conditions supporting linear growth of one stationary mode alone, results not presented here indicate that both travelling and other stationary modes may become linearly unstable at different parameters. From the point of view of maintenance of laminar flow by instability control, the existence of the new modes suggests that engineering methods of control of small-amplitude disturbances that aim at the frequencies of TS-like disturbances - as delivered by DNS or PSE and well approximated by an OSE solution-will have no impact on the global modes; the frequencies of the latter also have to be identified. However, a control method based on frequency information alone is bound to fail altogether if the unstable global mode is a stationary disturbance. A further significant result related to the new global modes concerns past conjectures that used topological arguments in an attempt to explain the origins of unsteadiness and three dimensionality of laminar separated flow. A linear mechanism is found and presented which, on account of the instability of the global mode and its spanwise periodicity, respectively, may lead to unsteadiness and three dimensionality in line with a multitude of experimental observations (see, for example, Dovgal et al. 1994). Being solutions of the equations of motion, the global modes should be reproducible by spatial DNS; on the other hand, the wave-like ansatz made in the PSE may question the applicability of the latter approach in identifying the PEPS modes; both questions are currently under investigation.

This paper is devoted to the memory of our colleague and friend Horst Bestek. Discussions with Ulrich Rist as well as his DNS data are appreciated.

\section{References}

Allen, T. \& Riley, N. 1995 Absolute and convective instabilities in separation bubbles. Aero. $J$. 99, 439-448.

Bertolotti, F. P., Herbert, Th. \& Spalart, P. R. 1992 Linear and nonlinear stability of the Blasius boundary layer. J. Fluid Mech. 242, 441-474.

Bestek, H., Gruber, K. \& Fasel, H. 1989 Self-excited unsteadiness of laminar separation bubbles caused by natural transition. In Proc. Conf. on the Prediction and Exploitation of Separated Flows, 18-20 October 1989. The Royal Aeronautical Society.

Briley, W. R. 1971 A numerical study of laminar separation bubbles using the Navier-Stokes equations. J. Fluid Mech. 47, 713-736.

Cebeci, T. \& Stewartson, K. 1983 On the calculation of separation bubbles. J. Fluid Mech. 133, $287-296$.

Dallmann, U. 1988 Three-dimensional vortex structures and vorticity topology. In Proc. IUTAM Symp. on Fundamental Aspects of Vortex Motion, Tokyo, Japan, pp. 183-189.

Dallmann, U., Herberg, Th., Gebing, H., Su, W.-H. \& Zhang, H.-Q. 1995 Flow field diagnostics: topological flow changes and spatio-temporal flow structures. AIAA 33rd Aerospace Sciences Meeting and Exhibit, AIAA-95-0791.

Dallmann, U., Vollmers, H., Su, W.-H. \& Zhang, H.-Q. 1997 Flow topology and tomography for vortex identification in unsteady and in three-dimensional flows. In Proc. IUTAM Symp. on Simulation and Identification of Organized Structures in Flows, Lyngby, Denmark, 25-29 May $199 \%$. 
Dovgal, A. V., Kozlov, V. V. \& Michalke, A. 1994 Laminar boundary layer separation: instability and associated phenomena. Prog. Aerospace Sci. 30, 61-94.

Hein, S., Bertolotti, F. P., Simen, M., Hanifi, A. \& Henningson, D. 1994 Linear nonlocal instability analysis - the linear NOLOT code. Internal report DLR IB 223-94 A56.

Hein, S., Theofilis, V. \& Dallmann, U. 1998 Unsteadiness and three-dimensionality of steady twodimensional laminar separation bubbles as result of linear instability mechanisms. Internal report DLR IB 223-98 A39.

Herbert, Th. 1997 Parabolized stability equations. A. Rev. Fluid Mech. 29, 245-283.

Howarth, L. 1938 On the solution of the laminar boundary layer equations Proc. R. Soc. Lond. A 164, 547-579.

Lin, R.-S. \& Malik, M. R. 1996 On the stability of the attachment-line boundary layers. Part 1. The incompressible swept Hiemenz flow. J. Fluid Mech. 311, 239-255.

Pauley, L. L., Moin, P. \& Reynolds, W. C. 1990 The structure of two-dimensional separation. J. Fluid Mech. 220, 397-411.

Rist, U. \& Maucher, U. 1994 Direct numerical simulation of 2-D and 3-D instability waves in a laminar separation bubble. In $A G A R D-C P-551$, Application of Direct and Large Eddy Simulation to Transition and Turbulence, pp. 34-1-34-7.

Rist, U., Maucher, U. \& Wagner, S. 1996 Direct numerical simulation of some fundamental problems related to transition in laminar separation bubbles. In Proc. Computational Fluid Dynamics Conf. ECCOMAS'96, pp. 319-325.

Schlichting, H. 1979 Boundary-layer theory. McGraw-Hill.

Spalart, P. R. \& Coleman, G. N. 1997 Numerical study of a separation bubble with heat transfer. Eur. J. Mech. B 16, 169-189.

Theofilis, V. 1997 On the verification and extension of the Görtler-Hämmerlin assumption in three-dimensional incompressible swept attachment-line boundary layer flow. Internal report DLR IB 223-97 A44.

Theofilis, V. 1998 Linear instability in two spatial dimensions. In Proc. Computational Fluid Dynamics Conf. ECCOMAS'98, Athens, Greece, 7-11 September 1998, pp. 547-552.

Theofilis, V. 2000 Globally unstable flows in open cavities. AIAA paper no. 2000-1965. 